\title{
DIVERSIDAD DE ESPECIES ALIMENTICIAS EN TRES MERCADOS AGRÍCOLAS DE LA HABANA, CUBA ${ }^{1}$
}

\author{
Tomás Shagarodsky², Víctor Fuentes ${ }^{3}$, Odalys Barrios ${ }^{2}$, Leonor Castiñeiras ${ }^{2}$, Zoila Fundora ${ }^{2}$, Pedro Sánchez ${ }^{2}$, \\ Lianne Fernández ${ }^{2}$, Raúl Cristóbal ${ }^{2}$, Maritza García $^{4}$, Celerina Giraudy ${ }^{5}$
}

\begin{abstract}
RESUMEN
Diversidad de especies alimenticias en tres mercados agrícolas de la Habana, Cuba. Se realizó el inventario de la diversidad de especies vegetales presentes en el mercado de la Habana, Cuba, para mostrar el comportamiento de tres especies modelo: el mamey colorado o sapote (Pouteria sapota (Jacq.) H. E. Moore et Stearn, especies del género Capsicum y el frijol caballero (Phaseolus lunatus L.), en mercados de diferentes dimensiones, los cuales reciben productos agrícolas de localidades de todo el país. En las especies modelos se evaluaron su frecuencia de aparición, precio, procedencia, y otras características. Hubo hasta 74 especies ofertadas en un mismo día, de las cuales se aprecian numerosos cultivares tradicionales de granos, frutas y hortalizas entre otros usos. La cuantía de lo inventariado permite detectar en el transcurso de 12 meses de evaluación unas 276 especies comprendidas dentro de 84 familias. Se incluyen en el estudio las viandas, los vegetales, medicinales, granos, frutales y ornamentales. Se aprecia al comparar con los productos observados en estudios realizados en huertos caseros de todo el país, que sólo una pequeña parte de la diversidad apreciada en ellos llega al mercado.
\end{abstract}

\begin{abstract}
Diversity of food species in three agricultural markets in Habana, Cuba. The inventory was conducted on the diversity of vegetable species present in the market and to show the behavior of three species in markets of different dimensions in the City of the Habana, which receive agricultural products from the whole country. The species used as model were: the red mammee or sapote (Pouteria sapota (Jacq.) H. E. Moore et Stearn, species of the gender Capsicum and the Lima bean (Phaseolus lunatus L.). In the key species, their availability frequency, price, origin, and other characteristics were evaluated. The inventory shows a great wealth that is manifested in occasions of up to 74 species offered in one day, of which numerous traditional cultivars of grains, fruits and vegetables are appreciated among other uses. The quantity of that inventory allowed to detect about 276 species within 84 families, in the course of 12 months of evaluation. The study included the roots and tubers, vegetables, medicinal, grains, fruits and ornamental plants. When comparing with the products observed in studies conducted in home gardens around the country, only a small part of this diversity reaches the market.
\end{abstract}

\section{INTRODUCCIÓN}

Los mercados pueden tener repercusiones tanto favorables como desfavorables sobre la biodiversidad y las culturas locales. La posibilidad de generación de ingresos para los miembros locales de la comunidad es potencialmente positiva. Sin embargo, la comercialización de los productos localmente disponibles no asegu- ra que las comunidades locales se beneficiarán de una manera equitativa y sostenible (Fries 2001). Entre los elementos que se deben tener en cuenta a la hora de desarrollar una estrategia de conservación para los recursos fitogenéticos in situ, está la posibilidad de que los productos o especies de interés agrícola que se conservan dentro de los huertos caseros y fincas de campesinos consigan potenciar su uso, por lo que sí dichos

1 Recibido para publicación el 15 de noviembre del 2002. Presentado en la XLVIII Reunión del PCCMCA. República Dominicana, 2002.

2 Instituto de Investigaciones Fundamentales en Agricultura Tropical "Alejandro de Humboldt" (INIFAT). E-mail: inifat@ceniai.inf.cu.

3 Instituto de Investigaciones en Fruticultura Tropical, Ministerio de Agricultura, Cuba.

4 Estación Ecológica Sierra del Rosario, Pinar del Río. CITMA Pinar del Río.

5 Áreas Protegidas Guantánamo, CITMA, Guantánamo. 
recursos alcanzan a tener una salida comercial en el mercado, con ello se puede lograr el apoyo a la conservación de la diversidad de cultivares tradicionales y especies que allí se atesoran.

El mercado puede ser un indicador de especies que existen en un país, cuáles poseen un interés de consumo y en consecuencia desarrollar estrategias para las especies que no llegan al mercado, incentivar su uso y contribuir a su conservación. Existen reportes de especies detectadas por primera vez a partir de colectas realizadas en el mercado como es el caso pino piñonero (Pinus maximartinezii Rzedowski) en México el cual representa un importante renglón económico para las familias campesinas propietarias de las tierras donde es endémica esta especie (Martin 2000).

El presente estudio se inició como parte del proyecto global "Contribución de los huertos caseros a la conservación in situ de recursos fitogenéticos en sistemas de agricultura tradicional" (Castiñeiras et al. 2000) donde se identificaron especies principales para el desarrollo de un estudio de diversidad las cuales fueron: el frijol caballero Phaseolus lunatus L., los ajíes y pimientos representado por las especies del Complejo del género Capsicum (C. annuum L., Capsicum chinense L. y $C$. frutescens L.) y el mamey colorado o zapote Pouteria sapota (Jacq.) Moore \& Stearn. De manera paralela se desarrolló una investigación a cerca del comportamiento de estas especies en relación al mercado, con el propósito de identificar la diversidad existente en los huertos familiares y en el mercado y establecer comparaciones entre ambos.(Shagarodsky et al., 2001 a, Shagarodsky et al 2001 b). Durante la ejecución del trabajo se observó que había una necesidad de inventariar no sólo las especies principales sino que se requería realizar el inventario de todo los productos que se expendían en el mercado por lo que se decidió extender el trabajo a la compilación de todas las especies.

Debemos destacar que los estudios de mercado son muy escasos en Cuba (Fernández et al. 1999; Shagarodsky et al, 2001) y la mayor parte de los artículos realizados en el país están dirigidos a plantas medicinales (Fuentes y Granda, 1988; Hernández Cano 1988 y Martínez Cándida 1990).

La existencia desde 1993 de los mercados agropecuario privados en Cuba ha posibilitado la existencia de un lugar para la inserción de una mayor diversidad de productos y aunque los precios son superiores a los existentes en la red comercial minorista, también ha permitido que los productos ganen en calidad (más frescos, transportados en menor tiempo y manipulados en menores volúmenes). Otras formas de mercado han aparecido como son los Mercados Agropecuarios Esta- tales que limitan en cuanto al precio que puede alcanzar el producto o el mercado paralelo.

El presente trabajo tiene como objetivo realizar el inventario general de la diversidad de especies presentes en el mercado y evaluar cómo las especies seleccionadas: mamey colorado, ajíes y frijol caballero se han distribuido durante un año de estudio en tres mercados de Ciudad de la Habana, considerando que en los mercados de las comunidades locales existe una baja frecuencia de estas especies. Estos productos sí están representados en los mercados de la capital, a pesar de originarse en muchas ocasiones en huertos caseros de los lugares más disímiles del país.

\section{MATERIALES Y MÉTODOS}

El trabajo se desarrolló en tres mercados de La Habana durante el período abril del 2001 a marzo del 2002. Los mercados estudiados fueron: Compostela de tamaño pequeño con un promedio anual de 28,4 tarimas o puestos; Egido, de tamaño intermedio con promedio anual de 46,8 tarimas y el mercado de Cuatro Camino considerado como grande por presentar un promedio anual de 85,94 tarimas.

Los mercados fueron visitados sábados, domingos o días feriados durante el 2001 y parte del 2002 para un promedio de más de tres muestreos por mes. Se tomó el número de tarimas que ofertaban el producto estudiado, teniendo presente la diversidad dentro de cada especie, de forma particular se evaluó como se distribuían tres especies modelos: el mamey colorado o zapote, los ajíes y pimientos y el frijol caballero. Adicionalmente, se estimó el porcentaje de tarimas que presentaban el producto con relación al número total presente en cada mercado.

La toma de muestra se realizó al menos dos veces por mes en el horario comprendido entre 10:00 am y 2:00 pm. La información se tomó mediante observación directa del producto en las tarimas, las tablillas que muestran el producto que se oferta y mediante entrevista a los vendedores. Se trató de confirmar que el producto que se expendía correspondía con lo que se mostraba en la tablilla pues con frecuencia no ocurría así, por desconocimiento o descuido del propio vendedor.

Se realizó un registro del número de formas vendidas, tipo de envases empleados y precios.

Se inventarió el total de especies presentes en los mercados estudiados mediante un listado de especies registradas. Dicho inventario se inició en abril del 2001 
hasta marzo del 2002. Conjuntamente con la información de los mercados citados, se evaluó ocasionalmente la presencia de especies en otros mercados de Ciudad de la Habana localizados en Santiago de las Vegas y Alamar (ambos de tamaño mediano) con el objetivo de comprobar si correspondía con las especies registradas para los mercados estudiados.

\section{RESULTADOS Y DISCUSIÓN}

\section{Especies presentes en el mercado}

El número máximo de especies por mes fue de 74 en el mercado grande, correspondiendo dicha cantidad de especies al mes de marzo en el cual se produce la cosecha de la mayoría de las especies hortícolas, granos y frutas; este mes también fue el que mayor número presentó tanto en el mercado pequeño como en el mediano con 58 y 66 especies respectivamente.

Se puso de manifiesto cómo el número de tarimas por mercado permite reafirmar la caracterización de los mercados estudiados como pequeño, mediano y grande debido a que se observaron claras diferencias en el número de puestos que expenden productos a pesar de que estos en cada muestreo eran variables. De igual forma se apreció que había claras diferencias entre la cantidad de especies que se presentaban en cada mercado, favoreciendo siempre dicha cantidad al mercado grande que difería claramente del pequeño y que siempre presentaba un número ligeramente mayor que el mediano.

Existen productos que a través de nuestro inventario (Cuadro 1), manifiestan su presencia en el mercado durante todo el año, está el caso de los plátanos en sus formas de fruta y verdura. Por lo general el plátano fruta tiene un precio mayor cuando está maduro y posee buen tamaño. Su forma de venta es en forma de mano principalmente en los tipos burro; y los tipos fruta y plátanos machos en forma de frutos individuales.

Durante todo el año, fue posible observar el coco de agua, a veces con la presencia de variedades perfectamente distinguibles por su tamaño como el coco de Baracoa; el culantro Eryngium foetidum, la fruta bomba o papaya, el pepino, la yuca, la habichuela china, el girasol, el frijol chino, los frijoles comunes en su variante negro, colorado y blanco, el ají Chay, ají cachucha, la calabaza, la albahaca, el arroz, la azucena, la berenjena, el boniato con varias variedades, cebolla, espárrago, extraña rosa, ajo de montaña, cebollino, los gladiolos de color Rosado, el mamey colorado, quimbombó, girasol, guayaba, maíz, col de repollo, frijol chino, naranja dulce, maní, margarita japonesa y yuca (Cuadro 1).

\section{Especies exóticas}

Hubo presencia en el mercado de especies exóticas, lo que manifiesta el interés de los campesinos por experimentar e introducir nuevas especies y variedades como el yatobá o kaki (Dyospirus kaki), los melocotoneros (Prunus persica) y las uvas de mesa (Vitis vinife$\mathrm{ra}$ ), todos ellos de regiones templadas, los cuales en algunos casos son cultivados en microclimas especiales o se han adaptado a nuestras condiciones, como es el caso de la uva de mesa en la localidad de Güira de Melena, provincia Habana. Otras especies tropicales también se ofertan en el mercado y que no tienen una presencia continuada, como son la ciruela china o carambola (Averrhoa carambola), mamoncillo chino (Litchi chinensis), la ciruela (Spondia purpurea). Otras frutas como el maracuyá (Passiflora edilis) fue observada con una baja frecuencia. Por lo general las especies citadas se distribuyen en una época muy específica y luego no vuelven aparecer hasta la próxima temporada o de acuerdo a su aceptación mantienen esta distribución estacional o simplemente no vuelve a ofertarse.

\section{Ornamentales}

El mercado de las especies ornamentales ocupa un espacio aparte pues este grupo ha ido en aumento en los últimos años la preferencia de los consumidores para cubrir sus necesidades espirituales y de otra índole que van desde diferentes festividades hasta su uso en actividades religiosas, de cumpleaños o sociales. Existen fechas muy señaladas donde la venta de flores es mayor (es el caso del día de las Madres, segundo domingo de mayo; día del Maestro, 22 de diciembre; el día 7 de diciembre (San Lázaro), de la patrona de Cuba: la Virgen de la Caridad, el día 8 de septiembre, o simplemente un ramo para una boda). Así el número de especies ornamentales se acrecienta e incluye tanto formas nativas o naturalizadas como especies exóticas. Son atractivas por la fragancia de sus flores las mariposas (Hedychium coronarium) y las azucenas (Polyanthes tubero$s a$ ) o por la variedad de formas y colores como las rosas (príncipe, blanca, miniatura, amarilla, etc).

Se detectaron también especies aromáticas como la albahaca (Ocimum spp) y un grupo de especies que sirven de soporte para los ramos que van desde los clásicos como la muraya (Murraya paniculata) y el esparrago (Asparagus spp). Otras no son tan comunes como Monstera deliciosa, el follaje de la propia mariposa, la 
Cuadro 1. Relación de especies y variedades comestibles y ornamentales observadas durante el estudio del mercado.

\begin{tabular}{|c|c|c|c|}
\hline Nombre común & Nombre científico & Meses & Familia \\
\hline Acelga china & $\begin{array}{l}\text { Brassica rapa } \mathrm{L} \text {. subsp. } \\
\text { chinensis (L.) Hanelt }\end{array}$ & $1,2,3,4,5,6,8,9,10,11,12$ & Brassicaceae \\
\hline Acelga española & Beta vulgaris var. cicla L. & $1,3,4,12$ & Chenopodiaceae \\
\hline Aguacate & Persea americana Mill. & $1,2,3,4,5,6,7,8,9,10,11$ & Lauraceae \\
\hline Ají arroz con pollo & Capsicum chinense Jacq. & 3 & Solanaceae \\
\hline Ají Cachucha & Capsicum chinense Jacq. & Todo el año & Solanaceae \\
\hline Ají Chay & Capsicum аппиит L: & Todo el año & Solanaceae \\
\hline Ají picante & Capsicum frutescens $\mathrm{L}$. & $1,2,3,6,7,8,9,10$ & Solanaceae \\
\hline Ajo & Allium sativum $\mathrm{L}$. & Todo el año & Alliaceae \\
\hline Ajo chino o ajo de montaña & Allium chinense G. Don. & Todo el año & Alliaceae \\
\hline Ajo porro & Allium porrum $\mathrm{L}$. & 4,7 & Alliaceae \\
\hline Ajonjolí & Sesamum orientale L. & $1,2,6,7,8,9,10,11,12$ & Pedaliaceae \\
\hline Albahaca & Ocimum basilicum L. & Todo el año & Lamiaceae \\
\hline Anón de ojo & Annona squamosa $\mathrm{L}$. & $8,9,10,12$ & Annonaceae \\
\hline Apio & Apium graveolens $\mathrm{L}$. & $1,2,3,4,5,6,7,11$ & Apiaceae \\
\hline Areca & $\begin{array}{l}\text { Dypsis lutescens (H. Wendl. } \\
\text { Beentje et J. Dransf.) }\end{array}$ & $3,4,5,7,8,9,10,11$ & Arecaceae \\
\hline Arroz & Oryza sativa $\mathrm{L}$. & Todo el año & Poaceae \\
\hline Ave del paraíso & Strelitzia reginae Ait. & 8,9 & Strelitziaceae \\
\hline Azucena & Polianthes tuberosa $\mathrm{L}$. & Todo el año & Agavaceae \\
\hline Berenjena & Solanum melongena $\mathrm{L}$. & Todo el año & Solanaceae \\
\hline Berro & $\begin{array}{l}\text { Rorippa nasturtium } \\
\text { aquaiticum (L.) Hayeek }\end{array}$ & $1,2,3,, 6,7,9,10,11$ & Brassicaceae \\
\hline Boca de león & Antirrhinum majus L. & $1,2,3,4,5,6$ & Scrophulariacea \\
\hline Boniato & Ipomoea batatas (L.) Lam. & Todo el año & Convolvulaceae \\
\hline Caimito Blanco & Chrysophyllum cainito $\mathrm{L}$. & $2,3,4$ & Sapotaceae \\
\hline Caimito morado & Chrysophyllum cainito L & 3,4 & Sapotaceae \\
\hline Cajigal & Zinnia elegans Jacq. & 9 & Asteraceae \\
\hline Calabaza & $\begin{array}{l}\text { Cucurbita moschata (Duch } \\
\text { ex Lam) Duch ex Poir. }\end{array}$ & Todo el año & Cucurbitaceae \\
\hline Canistel & $\begin{array}{l}\text { Pouteria campechiana (HBK) } \\
\text { Baehni }\end{array}$ & $1,2,3,4,8,9,10,11,12$ & Sapotaceae \\
\hline Cañandonga & Cassia grandis L. f. & $4,5,7$ & Caesalpinaceae \\
\hline Carambola & Averrhoa carambola $\mathrm{L}$. & $1,2,3,9,10$ & Oxalidaceae \\
\hline Cebolla amarilla & Allium сера & Todo el año & Alliaceae \\
\hline Cebolla corojo, de diente & $\begin{array}{l}\text { Allium cepa L. var. agregatum } \\
\text { G. Don. }\end{array}$ & $7,8,9,10,11,12$ & Alliaceae \\
\hline Cebolla roja & Allium cepa $\mathrm{L}$. & Todo el año & Alliaceae \\
\hline Cebollino & Allium fistulosum $\mathrm{L}$. & Todo el año & Alliaceae \\
\hline Cebollino & $\begin{array}{l}\text { Allium tuberosum Rottler } \\
\text { ex Spreng }\end{array}$ & $1,2,3,6,7,11,12$ & Alliaceae \\
\hline Chicharo & Pisum sativum $\mathrm{L}$. & 5 & Fabaceae \\
\hline Chayote blanco & Sechium edule (Jacq.)Sw. & $1,2,4,5,8,12$ & Cucurbitaceae \\
\hline Chirimoya & Annona reticulata $\mathrm{L}$. & $1,2,3,4,5,8$ & Annonaceae \\
\hline Cilantro de Castilla & Coriandrum sativum $\mathrm{L}$. & $1,3,9,10,11$ & Apiaceae \\
\hline Ciruela & Spondias purpurea $\mathrm{L}$. & 8,9 & Anacardiaceae \\
\hline Clavel Chino & Dianthus chinensis L. & $1,2,3,4,5,6$ & Caryophyllaceae \\
\hline Clavel español & Dianthus cariophyllus L. & $1,2,3,4,5,6,7,12$ & Caryophyllaceae \\
\hline Coco de Agua & Cocos nucifera $\mathrm{L}$. & Todo el año & Arecaceae \\
\hline Col de repollo & $\begin{array}{l}\text { Brassica oleracea L. var. } \\
\text { capitata L. }\end{array}$ & Todo el año & Brassicaceae \\
\hline Copal & Schinus terebentifolius Raddi & $3,4,5,6,7,8,9,10$ & Anacardiaceae \\
\hline Croto & $\begin{array}{l}\text { Codiaeum variegatum }(\mathrm{L} .) \\
\text { Blume }\end{array}$ & $3,9,10,11$ & Euphorbiaceae \\
\hline Culantro & Eryngium foetidum $\mathrm{L}$. & Todo el año, & Apiaceae \\
\hline Dalia decorativa formal & Dahlia pinnata Cav. & $1,2,3,4,5,7,9,10,11,12$ & Asteraceae \\
\hline Dalia cactus & Dahlia pinnata Cav. & $1,2,3,12$ & Asteraceae \\
\hline Dalia semidoble & Dahlia pinnata Cav. & $1,2,3,4,12$ & Asteraceae \\
\hline Encaje de Reina & Daucus carota L. & $3,4,5$ & Apiaceae \\
\hline Espada & Heliconia caribaea Lam. & $3,4,5,6$ & Heliconiaceae \\
\hline Espárrago & Asparagus aethiopicus L. & Todo el año & Asparagaceae \\
\hline Espinaca de Baracoa & $\begin{array}{l}\text { Talinum paniculatum (Jacq.) } \\
\text { Gaertn. }\end{array}$ & $1,2,3,8,9,12$ & Aizoaceae \\
\hline Espinaca de Malabar & Basella alba $\mathrm{L}$ & $1,2,3,4,5,6,7,8,9,10$ & Basellaceae \\
\hline
\end{tabular}


Continúa Cuadro $1 . .$.

\begin{tabular}{|c|c|c|c|}
\hline Nombre común & Nombre científico & Meses & Familia \\
\hline Extraña rosa & Calistephus hortensis Cass & Todo el año & Asteraceae \\
\hline Frescura & Pilea microphylla (L.) Liebm. & 1,2 & Urticaceae \\
\hline Frijol bayo & Phaseolus vulgaris L. & Todo el año & Fabaceae \\
\hline Frijol Blanco & Phaseolus vulgaris $\mathrm{L}$. & Todo el año & Fabaceae \\
\hline Frijol caballero & Phaseolus lunatus L. & $5,6,7$ & Fabaceae \\
\hline Frijol carita & Vigna unguiculata (L.) Walp. & $1,2,3,4,5,6,7,8,9,12$ & Fabaceae \\
\hline Frijol chino & Vigna radiata Roxb. & Todo el año & Fabaceae \\
\hline Frijol colorado & Phaseolus vulgaris L. & Todo el año & Fabaceae \\
\hline Frijol negro & Phaseolus vulgaris L. & Todo el año & Fabaceae \\
\hline Frijol de mantequilla & Phaseolus vulgaris L. & 7 & Fabaceae \\
\hline Fruta bomba & Carica papaya $\mathrm{L}$. & Todo el año & Caricaceae \\
\hline Garbanzo & Cicer arietinum $\mathrm{L}$. & $3,4,5,6,7,8,9,10,12$ & Fabaceae \\
\hline Gengibre o Agengibre & Zingiber officinale Roscoe & $1,2,3,4,5$ & Zingiberaceae \\
\hline Girasol & Heliathus аппииs L. & Todo el año & Asteraceae \\
\hline Gladiolo amarillo & Gladiolus communis $\mathrm{L}$. & $1,2,3,5,12$ & Iridaceae \\
\hline Gladiolo blanco & Gladiolus communis L. & $1,2,3,4,10,12$ & Iridaceae \\
\hline Gladiolo rojo (escarlata) & Gladiolus communis L. & $1,2,3,4,10,12$ & Iridaceae \\
\hline Gladiolo rosa & Gladiolus communis L. & $1,2,3,4,5,6,7,9,10,11,12$ & Iridaceae \\
\hline Gladiolo naranja & Gladiolus communis L. & $1,2,3,4,5,6,7,8,10,11,12$ & Iridaceae \\
\hline Gladiolo blanco con borde rosa & Gladiolus communis L. & 1,2 & Iridaceae \\
\hline Guanábana & Annona muricata $\mathrm{L}$. & $1,2,3,4,5,6,7,8,9,11,12$ & Annonaceae \\
\hline Guayaba & Psidium guajaba $\mathrm{L}$. & Todo el año & Myrtaceae \\
\hline Guacamaya francesa & Senna alata (L.) Roxb. & 9 & Caesalpinaceae \\
\hline Habichuela china & $\begin{array}{l}\text { Vigna unguiculata (L.) } \\
\text { Walp.cv-gr. sequipedalis (L.) } \\
\text { Verdc. }\end{array}$ & $1,2,3,4,5,6,7,8,9,11,12$ & Fabaceae \\
\hline Helecho & Nepholepis exaltata Schott & 7,9 & Polypodiaceae \\
\hline Helecho de cuero & Rumhora adiantiformis & $1,2,3,12$ & Polypodiaceae \\
\hline Hierba buena & Mentha spicata $\mathrm{L}$. & $3,5,6,8,9,10,11$ & Lamiaceae \\
\hline Leatriz & Liatris spicata Willd. & & Asteraceae \\
\hline Lechuga de hoja & Lactuca sativa $\mathrm{L}$. & $1,2,3,4,5,6,7,9,10,11,12$ & Asteraceae \\
\hline Limón criollo & Citrus aurantiifolia (Christm et & & \\
\hline & Panz.) Swingle & $1,2,3,5,6,7,8,9,10,11,12$ & Rutaceae \\
\hline Limón & Citrus limon Burm. $\mathrm{f}$ & $1,2,3,4,5,7,9,10,12$ & Rutaceae \\
\hline Lirio blanco & Crinum zeylanicum (L.) L. & $4,5,6$ & Amaryllidaceae \\
\hline Lirio rojo & Hymenocallis arenicola Northrop. & 4,6 & Amaryllidaceae \\
\hline Maíz grano & Zea mays L. & Todo el año & Poaceae \\
\hline Maíz harina & Zea mays $\mathrm{L}$. & Todo el año & Poaceae \\
\hline Maíz rosita & Zea mays $\mathrm{L}$. & $1,2,3,4,7,10,12$ & Poaceae \\
\hline Malanga Colocasia & $\begin{array}{l}\text { Colocasia esculenta (L.) Schott } \\
\text { et Endl. }\end{array}$ & $1,4,5,9,10,11,12$ & Araceae \\
\hline Malanga Xanthosoma Blanca & $\begin{array}{l}\text { Xanthosoma sagittifolium (L.) } \\
\text { Schott in Schott et Endl }\end{array}$ & $1,2,3,4,5,8,11,12$ & Araceae \\
\hline Malanga Xanthosoma Guaguí & $\begin{array}{l}\text { Xanthosoma sagittifolium (L.) } \\
\text { Schott in Schott et Endl }\end{array}$ & Todo el año & Araceae \\
\hline Mamey colorado & $\begin{array}{l}\text { Pouteria sapota (Jacq.) H.E. } \\
\text { Moore et Stearn. }\end{array}$ & Todo el año & Sapotaceae \\
\hline Mamoncillo & Meliccocus bijugatus Jacq. & $6,7,8,9,10$ & 58.Sapindaceae \\
\hline Mamoncillo chino & Litchi chinensis Sonn. & 4,12 & Sapindaceae \\
\hline Mandarina & Citrus reticulata Blanco & $1,2,3,4,9,10,11,12$ & Rutaceae \\
\hline Mango & Mangifera indica $\mathrm{L}$. & $3,4,5,6,7,9,11$ & Anacardaceae \\
\hline Manga Blanca & Mangifera indica $\mathrm{L}$. & 5 & Anacardaceae \\
\hline Mango de Clase & Mangifera indica $\mathrm{L}$. & $4,5,6,7,8,9$ & Anacardaceae \\
\hline Mango Toledo & Mangifera indica $\mathrm{L}$. & 9, & Anacardaceae \\
\hline Mango biscochuelo & Mangifera indica $\mathrm{L}$. & $4,5,6,8$ & Anacardaceae \\
\hline Mango Filipino & Mangifera indica $\mathrm{L}$. & 6 & Anacardaceae \\
\hline Maní crema & Arachis hypogaea $\mathrm{L}$. & Todo el año & Fabaceae \\
\hline Maní rojo & Arachis hypogaea $\mathrm{L}$. & Todo año & Fabaceae \\
\hline Maracuyá & $\begin{array}{l}\text { Passiflora edulis Sims var. } \\
\text { flavicarpa Degener }\end{array}$ & 6 & Passifloraceae \\
\hline Margarita japonesa & Gerbera jamesoni Hook & Todo el año & Asteraceae \\
\hline Mariposa (Flor) & $\begin{array}{l}\text { Hedichyum coronarium Koen in } \\
\text { Ritz }\end{array}$ & $6,7,8,9,10,11,12$ & Zigiberaceae \\
\hline Mariposa (hojas) & $\begin{array}{l}\text { Hedichyum coronarium Koen in } \\
\text { Ritz }\end{array}$ & $1,2,3,5,6,12$ & Zingiberaceae \\
\hline
\end{tabular}


Continúa Cuadro $1 .$.

\begin{tabular}{|c|c|c|c|}
\hline Nombre común & Nombre científico & Meses & Familia \\
\hline \multirow[t]{2}{*}{ Mariposa amarilla } & \multicolumn{3}{|l|}{ Hedichyum gardnerianum } \\
\hline & Shepparder ex Ker-Gaud in Edu. & 11 & Zingiberaceae \\
\hline Marigold & Tagetes erecta $\mathrm{L}$. & $3,4,6,12$ & Asteraceae \\
\hline Melocotonero & Prunus persica $\mathrm{L}$. & 9 & Rosaceae \\
\hline Melon de Agua & $\begin{array}{l}\text { Citrullus lanatus (Thunb.) } \\
\text { Matsimur et Nakai }\end{array}$ & Todo el año & Cucurbitaceae \\
\hline Melon de Castilla & Cucumis melo L. & 5 & Cucurbitaceae \\
\hline Moyita & Chrysanthemum coronarium $\mathrm{L}$. & $1,6,7,8,9,10,11,12$ & Asteraceae \\
\hline Ceriman de México, Malanga & Monstera deliciosa Liben. & $1,9,10$ & Araceae \\
\hline Muraya & Murraya paniculata (L.) Jack & $1,2,3,4,5,6,8,9,10,11,12$ & Rutaceae \\
\hline Naranja Agria & Citrus aurantium $\mathrm{L}$. & $1,2,3,4,5,6,7,, 9,10,11,12$ & Rutaceae \\
\hline Naranja Dulce & Citrus sinensis (L) Osbeck & Todo el año & Rutaceae \\
\hline Name & Dioscorea alata $\mathrm{L}$. & $1,2,3,4,5,6,7,9,10,11,12$ & Discoreaceae \\
\hline Nardos & Polianthes tuberosa $\mathrm{L}$. & $8,9,11,12$ & Agavaceae \\
\hline Orquidea & $\begin{array}{l}\text { Catleya trianaei Lineen et } \\
\text { Reichb. f. }\end{array}$ & $1,5,9,12$ & Orchidaceae \\
\hline Pepino & Cucumis sativus $\mathrm{L}$. & Todo el año & Cucurbitaceae \\
\hline Pera & Sysigium malaccense (L.) Merr. & 3 & Myrtaceae \\
\hline Perejil & Pretoselinum crispum (Mill.) Nym. & $1,2,3,4,5,6,7,8,11$ & Apiaceae \\
\hline Pimiento CW & Capsicum аппиит $\mathrm{L}$. & $1,2,3,4,5,6,7,8,10,11,12$ & Solanaceae \\
\hline Pimiento Español & Capsicum аппиит $\mathrm{L}$. & $2,3,5,6,7,8,9,10,11$ & Solanaceae \\
\hline Pimiento Verano-1 & Capsicum аппиит $\mathrm{L}$. & $3,4,5,8,9$ & Solanaceae \\
\hline Pimiento Hib. Amarillo & Capsicum аппиит $\mathrm{L}$. & 8 & Solanaceae \\
\hline Piña & Ananas comosus (L.) Merr. & $1,2,3,4,5,6,8,9,10,11,12$ & Bromeliaceae \\
\hline Plátano burro & Musa sp. (ABB) & Todo el año & Musaceae \\
\hline Plátano indio & Musa acuminata Cola (AAA) & 1 & Musaceae \\
\hline Plátano fruta & Musa acuminata Cola (AAA) & Todo el año & Musaceae \\
\hline Plátano Macho & Musa (AAB) & Todo el año & Musaceae \\
\hline Plátano manzano & Musa (AAB) & $3,4,5,6,8,9,10,11,12$ & Musaceae \\
\hline Plátano Pelipita & Musa sp. & $1,2,3,4,5,11,12$ & Musaceae \\
\hline Plátano FIAH & Musa sp. & $3,4,5,6,7,8,11$ & Musaceae \\
\hline Plátano vietnamita & Musa sp. (AAB) & $5,6,7,9,10,11,12$ & Musaceae \\
\hline Principe & Rosa sp. & Todo el año & Rosaceae \\
\hline Quimbombó Clemson & $\begin{array}{l}\text { Abelmoschus esculentus (L.) } \\
\text { Moench }\end{array}$ & Todo el año & Malvaceae \\
\hline Quimbombó Criollo & $\begin{array}{l}\text { Abelmoschus esculentus (L.) } \\
\text { Moench }\end{array}$ & $1,2,, 3,4,5,6,7,8,9,12$ & Malvaceae \\
\hline Quimbombó Emerald & $\begin{array}{l}\text { Abelmoschus esculentus (L.) } \\
\text { Moench }\end{array}$ & $6,7,8,9,12$ & Malvaceae \\
\hline Rabanito & Raphanus sativus L. & $1,2,3,4,5,6,7,8,10,11,12$ & Brassicaceae \\
\hline Remolacha & Beta vulgaris L. var. vulgaris & $1,2,3,4,5,6,7,11,12$ & Chenopodiaceae \\
\hline Rosa amarilla & Rosa sp. & Todo el año & Rosaceae \\
\hline Rosa blanca & Rosa sp. & $1,2,3,5,6,7,8,9,10$ & Rosaceae \\
\hline Rosa bulgara & Rosa sp. & $4,6,10,11$ & Rosaceae \\
\hline Rosa miniatura roja & Rosa sp. & 9 & Rosaceae \\
\hline Rosa miniatura rosada & Rosa sp. & $2,3,4,5,6,7,9$ & Rosaceae \\
\hline Sapote o Nispero & Manilkara zapota (L.) van Royen. & $1,2,3,4,5,6,7,8,11,12$ & Sapotaceae \\
\hline Sorgo o Millo & Sorghum bicolor $\mathrm{L}$. & 12 & Poaceae \\
\hline Tamarindo & Tamarindus indica $\mathrm{L}$. & $3,4,5,6,7,8,10$ & Caesalpinacea \\
\hline Siempreviva (Flor de papel) & Helychrysum bracteatum Andr. & 11,12 & Asteraceae \\
\hline Terciopelo & Celosia argentea $\mathrm{L}$. & $1,2,3,4,5,6,7,8,9,12$ & Amaranthaceae \\
\hline Tomate de cocina & Lycopersicon esculentum Mill & $1,2,3,5,6,8,9$ & Solanaceae \\
\hline Tomate de cocina (placero) & $\begin{array}{l}\text { Lycopersicon esculentum Mill. } \\
\text { var. cerasiforme (Dun.) Alef. }\end{array}$ & $1,2,3,5,6$ & Solanaceae \\
\hline Tomate en conserva & Lycopersicon esculentum Mill. & $2,3,4,5,6,9,11$ & Solanaceae \\
\hline Tomate ensalada & Lycopersicon esculentum Mill. & $2,3,4,5,6,11,12$ & Solanaceae \\
\hline Uva & Vitis vinifera $\mathrm{L}$. & $6,7,8,9,11$ & Vitaceae \\
\hline Vicaria & Cataranthus roseus (L.) G. Don. & 2 & Apocinacia \\
\hline Yatobá & Dyospirus kaki Thunb. & 11 & Ebenaceae \\
\hline Yuca Blanca & Manihot esculenta Crantz & $2,3,5,7,10,11,12$ & Euphorbiacea \\
\hline Yuca CMC-40 & Manihot esculenta Crantz & Todo el año & Euphorbiacea \\
\hline Zanahoria & Daucus carota $\mathrm{L}$. & $1,2,3,4,5,6,11,12$ & Apiaceae \\
\hline
\end{tabular}


areca (Dypsis lutescens), el croto (Codiaeum variegatum), el copal (Schinus terbentifolius) u hojas de palma del género Thrinax. También se utilizan como soporte de los ramos la propia estructura de la planta como es el caso de los gladiolos (Gladiolus communis) que presentan múltiples colores que van desde el blanco hasta el rojo escarlata pasando por el amarillo, naranja hasta el más típico de color rosa o el girasol (Helianthus annuиs) que es una especie de alta preferencia. Es muy común el uso como adorno de los ramos una umbelífera del género Daucus reconocida como Encaje de la reina. Las flores por lo general se acompañan de jarras, películas de nylon que las protege contra la deshidratación, cintas, o se le añade películas de colores para mejorar su atractivo.

En una fecha reciente se ha introducido en el mercado varias especies ornamentales que son producidas con cuidados especiales en zonas de montaña tal es el caso de la espiga conocida como Leatriz (Liatris spicata), y otras se importan y se venden en el mercado como adorno para los ramos de flores como es el caso del helecho de cuero Rumhora adianthiformis.

\section{Hortalizas y condimentos}

Se pone de manifiesto en el mercado el amplio empuje que ha tenido la producción de hortalizas en el país, influido principalmente por el Programa de Agricultura Urbana que ha permitido una oferta de este grupo de especies con un producto de mayor calidad (Rodríguez et al. 2002). Por lo general, las hortalizas de hojas llegan muy temprano y se terminan con prontitud, lo que hace que el inventario de dichas especies deba realizarse entre 10:00 am y 12:00 m o en su defecto observar la tablilla y confirmar con el vendedor. Resulta frecuente percibir la venta de quimbombó, lechugas, acelgas chinas, cebollinos, perejil, entre otras hortalizas. También dentro de este grupo se cuenta con la presencia de las espinacas de Malabar (Basella alba) o la llamada espinaca de Baracoa (Talinum paniculatum) que pone de manifiesto una mayor cultura en cuanto al consumo de hortalizas de hoja, cosa que no sucedía en décadas anteriores, donde no existía un mercado con las características actuales.

Se ofrecen también como hortaliza los brotes germinados del frijol chino o frijol mungo (Vigna radiata) y es constante la presencia del ajo y la cebolla. Esta última con precios elevados debido a su alta demanda. La cebolla se presenta antes de la temporada, expendiéndose sin realizar el cierre del cuello y con las hojas aún verdes, incluye tanto variedades comerciales importadas como las variedades tradicionales llamadas cebollas corojo o de diente (Allium cepa var aggregatum).
Entre los condimentos muchas de las especies que se expenden en el mercado no se encuentran directamente en las tarimas sino que por lo general se vende aparte por vendedores ambulantes e incluyen varias especies exóticas y naturalizadas entre las que se encuentran el laurel (Laurus nobilis), la canela (Cinnamomon aromaticum) en rama o en polvo, el orégano (Origanum vulgare), la pimienta negra (Piper nigrum), la nuez moscada (Myristica fragans), el comino (Cuminum cyminum), la pimienta dulce (Pimenta dioica) y la Bija (Bi$x a$ orellana) (Cuadro 2). Además se vende condimentos secos de ajo o cebolla deshidratado u otra forma conocida como sazonador que incluye además otras especies tales como orégano cimarrón (Ocimum gratissimum).

Cuadro 2. Condimentos secos vendidos en el mercado por vendedores ambulantes o vendedores con puestos establecidos.

\begin{tabular}{lll}
\hline Nombre vulgar & \multicolumn{1}{c}{ Nombre científico } & \multicolumn{1}{c}{ Observaciones } \\
\hline $\begin{array}{l}\text { Bija - Bijol } \\
\text { Sazonador }\end{array}$ & Bixa orellana & \\
& -- & $\begin{array}{l}\text { Mezclas de especies } \\
\text { que incluyen ajo, } \\
\text { cebolla, deshidratada }\end{array}$ \\
Pimienta dulce & Pimenta dioica & Hojas y frutos \\
Pimienta picante & Piper nigrum & Frutos secos \\
Orégano & Origanum vulgare & Se vende molido o \\
& & en grano \\
Orégano francés & Plecthranthus amboinicus & Hojas deshidratadas \\
Canela & Cinnamomon aromaticum & En rama o molida \\
Laurel & Laurus nobilis & Hoja entera o molido \\
Comino & Cuminum cyminum & Molido o en grano \\
Pimentón & Capsicum annum & Ligeramente picante \\
Nuez moscada & Myristica fragans & Raras veces observada \\
Orégano cimarrón & Ocimum gratissimun & -- \\
\hline
\end{tabular}

\section{Medicinales y especies de uso ceremonial o ritual}

A pesar de que en los mercados estudiados no había muchos puestos dedicados a la venta de especies medicinales y/o ceremoniales es precisamente este grupo el qué mayor número de especies aportó al inventario general. Se pudo determinar la presencia de 167 especies correspondientes a 69 familias lo que representa el 53,3\% de las especies citadas en este reporte. De estas especies 20 ya han sido señaladas en otras categorías de uso (Cuadro 1). En este grupo se destacan especies como el maíz que se utilizan sus estambres como diurético, la mazorca seca tostada para uso ceremonial, molido en forma de harina para alimento animal, tierno para consumo humano utilizado en la preparación de tamales y otros platos. En este grupo también están presente los cítricos y algunas anonáceas de los 
cuales se utilizan sus ramas y hojas en la preparación de té destinado al tratamiento de diversas afecciones. Se encontró en el puesto de venta de medicinales retoños de gandul (Cajanus cajan) utilizados como antiparasitario, sin embargo, esta especie no se registró como grano en el mercado, de igual forma se observó la presencia del mamey de Santo Domingo (Mammea americana) como medicinal, pero no apareció expendida como fruta. Se destacan como plantas medicinales por su alta demanda la manzanilla (Egletes villosa), el tilo (Justicia pectoralis), el romero (Bidens pilosa var radiata) entre otros y por su uso como ritual o ceremonial el coco (Cocos nucifera), la siguaraya (Trichilia glabra), vencedor (Vitex agnus-castus), albahaca (Ocimum basilicum).

\section{Confusión de nombres}

Resulta frecuente observar que cuando realizamos el inventario, varias especies son llamadas bajo un mismo nombre; así se denomina cebollino a varias especies y variedades de la familia Alliaceae como Allium fistulosum, A. tuberosum, A. porrum, y A. cepa var. agregatum, los cuales se reconocen en otros sitios del país como cebollino, ajo de montaña, ajo porro, y cebolla corojo o multiplicadora. En el caso de los granos es frecuente que se venda como frijol común (Phaseolus vulgaris) algunas variedades de frijol carita (Vigna unguiculata) o en el caso de los granos blancos se sustituya frijol caballero (Phaseolus lunatus) por frijol común. En el caso de los Allium resulta necesario que se esclarezca las denominaciones a fin de lograr una mejor concordancia entre la calidad, demanda y precios.

Otra confusión frecuente de los vendedores se encuentra cuando se llama cilantro a lo que comúnmente se denomina culantro (Eryngium foetidum) o viceversa, no correspondiendo con el Cilantro de Castilla (Coriandrum sativum). En realidad el nombre común no es patrimonio de una u otra especie y la confusión tiene justificación no en las características morfológicas de ambas especies que se diferencian claramente, sino en su olor y sabor.

\section{Calidad}

En cuanto a la calidad de los frutos que se vende, en muchas ocasiones, fue muy baja y aunque tratan de utilizar empaques de nylon $u$ otra modalidad, el producto se expone al sol en aquellos mercados al aire libre o tiene evidentes signo de virosis en frutos de ajíes y tomates o están deteriorados por la acción de la humedad o golpes que recibe durante su traslado, no obs- tante, no se modifican sus precios. Tampoco, se observan en un mismo día diferencias de precios entre un vendedor $u$ otro debido a que la demanda es muy superior a la oferta. En algunas frutas como el mamey colorado, los plátanos, las guayabas cuando están en franco deterioro y ya se aproxima el cierre del mercado o es fin de semana, entonces el vendedor toma la decisión de bajar su precio. Por lo general un alto precio es un indicio de una mayor calidad de lo que se vende. Sucede así en el caso de los mamey y las frutas en general.

En el caso de los ajíes es frecuente observar la venta de mezcla de variedades. Ocasionalmente se observan ajíes picantes dentro de formas vendidas como dulces. Otra especie en la que cada vez es más frecuente observar mezclas mecánicas, es el maní donde se vende de manera conjunta los granos rojos con los del tipo crema. Las variedades de maní del tipo crema se han popularizado como consecuencia de la inserción en la producción de variedades resultantes del mejoramiento genético.

\section{Estudio de las principales especies modelos y com- portamiento en el mercado}

\section{Pouteria sapota}

De manera general, la mayor abundancia del mamey se observó durante la primavera y el verano, aunque se notó el producto durante todo el año.

Los precios del fruto fueron variables y están asociados al tamaño de la fruta o a su abundancia, los precios variaron entre $\$ 1$ y $\$ 25$ pesos. Resulta interesante señalar que a veces en un mismo puesto hay más de una forma de fruto, pues por lo general el que vende esta fruta se especializa en su venta y acopia la misma una vez llegado al mercado, aunque cuando el producto abunda son muchos los vendedores que lo venden.

Un estudio previo de Pouteria sapota (Shagarodsky et al. 2000) determinó que los caracteres longitud y peso del fruto muestran alta variabilidad. El consumidor por lo general busca el color rojo-naranja con adecuado tamaño. Otro elemento que se tiene en cuenta es que la fruta esté madura y que no tenga Primavera (desorden fisiológico que provoca un endurecimiento de la pulpa del fruto o secciones de una parte de ella que invalidan a este para el consumo). En ocasiones se compra mamey colorado verde el cual es utilizado como ofrenda religiosa. Es poco común que se señale el uso de la semilla del mamey como cosmético, medicinal o en la alimentación animal (CONAFRU 1974, IPGRI-BID). 
Dada la demanda y el alto precio del mamey colorado, los propios vendedores señalan que una semana después de la cosecha es más que bueno para venta; corre el riesgo de perder la fruta durante el transporte y venta. El transporte se realiza por tren en cajas de madera; se vende tanto a consumidores directos como a personas que elaboran batidos en La Habana.

Otras especies de interés de la misma familia Sapotáceas dentro del mercado, sin llegar a tener la significación del mamey colorado, es el canistel Pouteria campechiana, el caimito Chrysophyllum cainito y el zapote o níspero Manilkara zapota. Estas especies tienen una distribución estacional más específica; el canistel ha sido observado durante los meses de marzo, agostodiciembre; el caimito se presenta en dos variedades morado o blanco en los meses de marzo y abril y el níspero el cual se presenta con variedades y tamaños diferentes se ha observado durante los meses de marzojunio, octubre- diciembre.

Al igual que con el mamey colorado, estas otras especies se venden como fruto verde para ofrendas religiosas.

\section{Capsicum spp.}

El mayor porcentaje lo presentó la especie Capsicum annuum, seguido de $C$. chinense y con muy baja frecuencia $C$. frutescens. Esto indica de manifiesto la importancia de los ajíes y pimiento como condimento y especie hortícola en Cuba. El predominio de C. annuum está relacionado con la alta representatividad de variedades comerciales en el mercado como son el ají Chay; el pimiento del tipo California Wonder y el pimiento Español; por lo general de estas formas se hace una distribución de semilla de estas variedades por el estado que se adquiere por los campesinos por diferentes vías. Se observó también la presencia de variedades de color amarillo y de un tamaño muy grande que manifiestan la presencia de semillas híbridas procedentes de casas comerciales extranjeras. El ají Chay constituye una forma que puede observarse durante todo el año aunque en nuestro caso su mayor frecuencia se apreció en el mes de octubre.

Las variedades tradicionales correspondientes a la especie $C$. chinense, como el ají cachucha, el arroz con pollo, se muestran con una menor frecuencia debido a que las mismas no son variedades comerciales y su producción se limita a propietarios privados, las cuales son altamente demandadas por su apreciable aroma y sabor como condimento fresco. En el caso del ají arroz con pollo, son tipos específicos que han sido colectados en las provincias de Holguín y Las Tunas y resulta muy probable que ésta fuera su procedencia debido a que sólo fueron observados una vez en el mes de marzo, aunque en un estudio anterior había sido observado en los meses de octubre y noviembre. Dentro de esta misma especie se observó luego de indagar de manera directa otra variedad conocida 'ají de mantequilla', y otras que denominan los vendedores del mercado de Santiago de las Vegas como 'Hemingway' y 'ají cachuchón' ambas de frutos acostillados y con un precio bastante elevado ( $25 \$$ / libra).

La especie $C$. frutescens se encontró con muy baja frecuencia en un mercado grande (Figura 1), siempre localizada en un puesto que distribuía otras especies de vegetales de hoja y a veces dentro de un puesto que ofertaba plantas medicinales dentro del mercado mediano. Se reconoce a estos tipos como 'ají guaguao' o 'ají picante' debido a su alta pungencia. Estas variedades son características de algunas comidas en Cuba, pero por lo general el picante no es típico en nuestra cocina tradicional, aunque algunos gustan de preparar con este un aliño para saborizar las comidas. El uso medicinal de esta especie ha sido referido en entrevistas realizadas a campesinos trabajos realizados por el equipo del proyecto Huertos Caseros (Fuentes et al. 2000). También se ha advertido en una ocasión ajíes del tipo Cayena los cuales son muy picantes, pero que pertenecen a la especie Capsicum annuum.

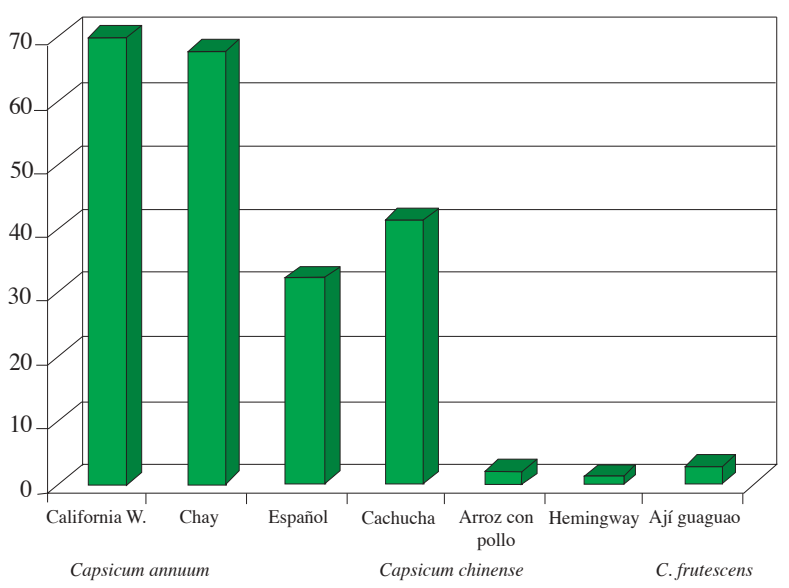

Figura 1. Frecuencias máximas observadas en variedades ají o pimiento en el estudio de tres mercados de Ciudad de la Habana, Cuba.

Hemos notado que la venta del ají guaguao se realiza al menudeo costando los frutos colocados en una taza de café un precio de $3 \$$ o la misma cantidad dentro de pequeños paquetes de nylon o papel durante los meses de enero-marzo y junio- octubre (Cuadro 3 ). 
En el caso de los ajíes se evidencia a través del mercado que las potencialidades de estas especies no han sido explotadas del todo y que los cultivares locales poseen valores aún no estudiados. Se conoce que en muchas ocasiones una buena parte de la producción se pierde y no llega al mercado producto de falta de transporte o simplemente porque no se tiene conocimiento de las diferentes formas de preparación, pues prácticamente la conserva de especies como el ají no existe y puede ser un elemento que puede contribuir a la economía del huerto casero (de manera deshidratada, encurtido, concentrada o fresco), con la activa participación de la mujer y redundando ello en el interés de los propios campesinos por la preservación in situ de la diversidad de recursos fitogenéticos presentes en los huertos. Se han desarrollado en la capital del país proyectos comunitarios que estimulan a la comunidad a fomentar la conservación de alimentos de manera diversa (Figueroa y Lama, s. a) aunque los mismos no se reflejan en el mercado de manera amplia sino por lo general sólo para productos de alta demanda como el tomate. No obstante, se ha observado otras especies conservadas como el tamarindo, se oferta coco molido o ajíes encurtidos.

\section{Phaseolus lunatus}

La presencia del frijol caballero en muy pocos casos se observó en el mercado, incluso en aquellos mercados locales como el de Palenque, Guantánamo (Shagarodsky et al. 2001 b), zona donde existe una gran diversidad para esta especie. La baja presencia en el mercado es una evidencia de que esta especie es propia de los huertos caseros y fuera de ellos es muy difícil encontrarla en plantaciones comerciales (Castiñeiras et al. 2001). En este estudio esta especie sólo fue observada en los meses de mayo, junio y julio presentadas como frijol común (Phaseolus vulgaris) en sus variantes de color blanco.

Durante el desarrollo de los programas de capacitación del proyecto Huertos Caseros en Guantánamo al- gunos campesinos señalaron que esta especie tenia una madurez no agrupada, lo que hacía que prácticamente no se pudiera comercializar. Sin embargo, con un adecuado manejo agronómico e incremento de las poblaciones de esta especie es posible desarrollar este renglón produciendo en una etapa en la que otros granos incluido el frijol común no se puede sembrar por las altas precipitaciones y temperaturas.

El frijol caballero tiene una pobre utilización en la actualidad y requiere una mayor atención, pues se ha perdido la costumbre de uso y se desconoce su forma de preparación. De forma general la población joven de zonas urbanas y de zonas rurales no conoce esta especie. A mediados del siglo XX esta especie tuvo una alta demanda como habas de lima que corresponden a Phaseolus lunatus cv.-gr 'Big Lima' llegando la misma a exportarse, sin embargo, aún cuando hay variedades comerciales de esta especie y en los huertos caseros de los campesinos se cultiva, se puede categorizar que este es un cultivo subutilizado. En este caso se impone la promoción de las potencialidades de este cultivo en el contexto local con fines de diversificación de la alimentación de las comunidades y promoción de la producción de leguminosas de granos nativas contribuyendo a su conservación.

\section{Comparación del mercado y los huertos caseros}

Se evidenció que una gran parte de las especies que hoy se encuentran en los huertos caseros del país no llegan al mercado, entiéndase tanto en el rango de especies como de variedades o clones pues la diversidad percibida en los huertos caseros es muy superior. Es cierto que muchas especies no cumplen las expectativas del mercado; sin embargo, resulta insuficiente el número de entidades cuyo potencial de comercialización hoy no se explotan. La vía de inserción en el mercado de muchos productos aún no se ha logrado asimilar por parte de las familias propietarias de los huertos, en

Cuadro 3. Frecuencias máximas observadas para los diferentes tipos de ajíes y pimientos.

\begin{tabular}{|c|c|c|c|c|}
\hline $\begin{array}{l}\text { Variedad } \\
\text { máximas }\end{array}$ & $\begin{array}{c}\text { Frecuencia } \\
\text { observadas } \\
(\%)\end{array}$ & $\begin{array}{l}\text { Mes de mayor } \\
\text { frecuencia }\end{array}$ & $\begin{array}{l}\text { Tipo de Mercado } \\
\text { Donde se observó }\end{array}$ & Especie \\
\hline California Wonder & 70,0 & Enero & Pequeño & Capsicum annuum \\
\hline Chay & 67,8 & Octubre & Pequeño & Capsicum аппиит \\
\hline Español & 32,1 & Junio & Pequeño & Capsicum аппиит \\
\hline Cachucha típico & 41,4 & Abril & Pequeño & Capsicum chinense \\
\hline Arroz con pollo & 1,8 & Marzo & Grande & Capsicum chinense \\
\hline Heminguay & 0,9 & Marzo & Grande & Capsicum chinense \\
\hline Ají guaguao & 2,6 & Marzo & Grande & Capsicum frutescens \\
\hline
\end{tabular}


muchos casos por desconocimiento y la falta de medios como el transporte, de envases, y otros recursos. Algunas especies pueden lograr su inserción en el mercado con la aplicación de procesamiento artesanal que le de valor agregado al producto y permita su conservación por un período más largo. Así en el caso de los ajíes (Capsicum spp) es posible trabajar la deshidratación de los tipos picantes, o la concentración de pulpa de los tipos dulces o la preparación de macetas que permitan la venta como decorativo a los llamados ajíes de jardín (Capsicum annuum). Otros productos de los huertos utilizados como condimentos en forma fresca tampoco son comercializados de manera sistemática como es el caso del oreganito (Lippia micromera) o el tomillo (Thymus vulgaris); la albahaca (Ocimum tenuiflorum). En el campo de los frutales mucho terreno queda por explotar trabajando en su preparación para el mercado y haciéndolas más atractivas para el consumidor entre ellos se encuentran algunos cítricos y sapotáceas.

Algunas especies típicas de los huertos caseros tienen una baja representatividad en el mercado o simplemente no están presentes; es el caso del chayote ( $\mathrm{Se}$ chium edule) o ninguna presencia del gandul (Cajanus cajan) como grano; la calabaza china (Benincasa hispida), el frijol mambí o frijol arroz (Vigna umbellata) e incluso el frijol caballero (Phaseolus lunatus). Ello pone de manifiesto que queda aún mucho por hacer en la diversificación de los productos del mercado agrícola en Cuba, de manera que pueda contribuir a ampliar la base alimentaria de los habitantes del país y en consecuencia su salud y bienestar.

El inventario general permitió apreciar la presencia de 154 formas comercializadas correspondientes a 113 especies, mostrando algunas especies una alta diversidad intraespecífica que corresponde con los patrones de consumo de nuestra población y el papel que juegan los mismos en la alimentación de la población, tal es el caso del frijol común y los plátanos (Musa spp). Entre las ornamentales presentaron una alta diversidad el género Rosa y Gladiolus. El mango (Mangifera indica) y el aguacate (Persea americana) se destacan por una significativa diversidad.

Al comparar estos resultados con los inventarios obtenidos en los huertos familiares ponen de manifiesto una gran diferencia entre lo que se comercializa y lo que realmente hay en los huertos familiares y fincas de campesinos. El inventario general realizado en tres regiones representativas del occidente, centro y oriente de Cuba como resultado del proyecto "Contribución de los huertos caseros a la conservación in situ de la diversi- dad de Recursos Fitogenéticos en Sistemas de Agricultura Tradicional" Componente: Cuba (Castiñeiras et al. 2002) arrojó un total de 508 especies de las cuales el $80 \%$ (406) son cultivadas. También como parte de este estudio fue posible determinar que sólo el $14 \%$ de las especies presente en el huerto llegaban a tener alguna forma de comercialización (Fundora et al. 2001).

El inventario de especies en el mercado muestra una gran riqueza, que se manifiesta en ocasiones en una cuantía de hasta 74 especies ofertadas en un mismo día, de las cuales se aprecian numerosos cultivares tradicionales de granos, frutas y hortalizas entre otros. La venta de una gran diversidad de productos en el mercado, muchos de ellos desconocidos para las generaciones más jóvenes, contribuye a que se conozca y se divulgue su forma de utilización y con ello a su conservación para las generaciones presentes y futuras, siempre que se haga de una forma adecuada.

\section{CONCLUSIONES Y RECOMENDACIONES}

La cuantía de lo inventariado permitió detectar en el transcurso de un año de evaluación unas 276 especies comprendidas dentro de 84 familias. De estas especies el 53,3\% se expenden como medicinales y/o rituales.

Del total de especies inventariado se venden como plantas comestibles, condimentos secos y ornamentales 130 especies de 51 familias.

Se observó dentro de las especies modelos, que el mamey colorado en el mercado se distribuyó durante todo año poniendo de manifiesto una alta diversidad.

Se observaron especies propias de los huertos caseros como es el frijol caballero (Phaseolus lunatus) las cuales se presentaron en el mercado con una baja frecuencia y en consecuencia se debe trabajar en el desarrollo de sus potencialidades para el mercado, buscando el fomento de la conservación a través del uso.

En el caso de los ajíes y pimientos se evidencia a través del mercado que las potencialidades de estas especies no ha sido explotadas del todo y que los cultivares locales poseen valores aún no explotados.

El mercado es un espacio al cual debe prestársele atención pues contribuye a la preservación de la diversidad a través del uso. 


\section{LITERATURA CITADA}

ADERALDO, G. F. 1999. Diversidad genética de la colección de sapote (Pouteria sapota (Jacquin) H. E. Moore y Stearn) del CATIE. Plant Genetic Resources Newsletter 117: $37-42$.

CASTIÑEIRAS, L., FUNDORA, Z.; SHAGARODSKY, T.;MORENO, V.;, O. BARRIOS, L. FERNÁNDEZ Y R. CRISTÓBAL. 2002. Contribution of Home gardens to in situ conservation of Plant Genetic Resources in Farming Systems - Cuban Component, Project reports. In: Watson J. W. and P.B. Eyzaguirre (eds.) Proceedings of the Second International Home Gardens Workshop: Contribution of Home gardens to in situ conservation of Plant Genetic Resources in Farming Systems, 17-19 July, 2001. Witzenhausen, Federal Republic of Germany. International Plant Genetic Resources Institute, Rome, p 42-55.

CASTIÑEIRAS, L., FUNDORA, Z.; SHAGARODSKY, T.; FUENTES, V.; BARRIOS, O.; MORENO, V.; SÁNCHEZ, P.; GONZÁLEZ, A. V.; MARTÍNEZ, A.; GARCÍA, M.; MARTÍNEZ, A. (2000) La conservación de la diversidad de plantas de cultivo en dos localidades de Cuba. Revista del Jardín Botánico Nacional vol. XXI (1): 25-45.

CASTIÑEIRAS, L., MORENO, V., SHAGARODSKY, T.; FUNDORA, Z.; BARRIOS, O.; FUENTES, V. R.; FERNÁNDEZ, L.; SÁNCHEZ, P.; CRISTÓBAL, R.; WALÓN, L.; PÉREZ, M. F. Y PÚLDÓN, G. 2001. El frijol caballero (Phaseolus lunatus L.), un cultivo marginal en peligro de erosión genética en Cuba. Revista del Jardín Botánico Nacional (Cuba) 22(1):133-138 .

CONAFRU (Comisión Nacional de Fruticultura, México). 1974. El cultivo del mamey. Serie Divulgación. Folleto No. 14, SAG/ México, 20 pp.

DANIELLS, J.; FRISON, E.; KARAMURA, D.; JENNY, C.; LESCO, T. ; SHARROCK, S. ; SWENNEN, R.; TONEKPEAND, K. Y SUBBARAYA, U. 2001. Musalogue: a catalogue of Musa Germplasm. Diversity in the genus Musa (E. Anaud and S. Sharrock, compil.) International Network for the Improvement of Banana and Plantain, Montpelier, Frace, 213 pp.

FERNÁNDEZ, M.; MARTÍNEZ, C.; ROSETE, S.; MARTÍNEZ, J. I.1999. Agromercados en Cuba: Reservorios de Germoplasma. Acta Botánica Cubana (Cuba) No. $125: 1-8$

FIGUEROA, V.; LAMA, J. (s.a) Del Patio Familiar a la cocina. Proyecto Comunitario: Conservación de Alimentos. Marianao, C. Habana, Cuba. 44 pp.

FRIES, A. M. 2001. La iniciativa de Yunnan: Propuestas internacionales sobre diversidad cultival y biológica. LEISA Revista de Agroecología. 17(2):33.
FUENTES, V., CRISTÓBAL, R.; SHAGARODSKY, T.; SÁNCHEZ, P.; CASTIÑEIRAS, L.; FUNDORA, Z.; BARRIOS, O.; MORENO, V.; FERNÁNDEZ, L.; ORELLANA, R.; ALONSO, J. L.; GONZÁLEZ, V.; GARCÍA, M.; GIRAUDY, C.; VALIENTE, A.; ROBAINA, R. 2000. Plantas medicinales en conucos de tres regiones de Cuba (2000) VII Encuentro de Botánica "Johannes Bisse in Memoriam". Camagüey, Instituto Superior Pedagógico "José Martî", 20 de diciembre del 2000, Cuba.

FUENTES, V.; GRANDA, M.1988. Estudios sobre la medicina tradicional en Cuba I. Revista Plantas Medicinales (Cuba) 2:25-46.

HERNÁNDEZ, J. 1990. Plantas que se expenden en las yerberías de la ciudad de Santiago de Cuba. Resúmenes II Simposio de Botánica. Ciudad de La Habana, 14-17 de junio de 1990.

IPGRI-BID. Diversidad; Conservación y uso sostenible de los Recursos Genéticos de Frutales Nativos de América Tropical. Informe Final. Cooperación Técnica IPGRI/BID No ATN/SF-4356-RG, 23 pp. IPGRI Oficina Regional para las Américas, Cali, Colombia.

MARTÍN, G J. 2000. Etnobotánica: Manual de métodos. Editorial Nordan-Comunidad. Montevideo, Uruguay, Fondo Mundial para la Naturaleza (WWF). pp.178-179.

MARTÍNEZ, C. 1990. Estudio etnobotánico de algunas yerberías de Ciudad de La Habana. Resúmenes V Congreso Latinoamericano de Botánica. Ciudad de la Habana, 24-29 de junio de 1990.

RODRÍGUEZ, A. 2002. La Agricultura Urbana en Cuba un aporte a la seguridad alimentaria. Grupo Nacional de Agricultura Urbana, Presentación realizada el 4 de octubre del 2002. Ministerio de Agricultura, Cuba.

SHAGARODSKY, T.; FUNDORA, Z.; CASTIÑEIRAS, L.; BARRIOS, O.; MORENO, V.; FERNÁNDEZ, L.; FUENTES, V. R., CRISTÓBAL, R., GIRAUDY, C., GARCÍA, M., SÁNCHEZ, P., GONZÁLEZ, V., HERNÁNDEZ, F. Y VALIENTE, A. (2001). Diversidad de productos agrícolas en los sistemas de fincas y su presencia en el mercado. Cuba. Agricultura Orgánica. Año 7 (1): 24-28.

SHAGARODSKY, T.; CASTIÑEIRAS, L.; FUENTES, V. R.; CRISTÓBAL, R.; FUNDORA, Z.; GIRAUDY, C.; GARCÍA, M.; SÁNCHEZ, P.; BARRIOS, O.; FERNÁNDEZ, L.; HERNÁNDEZ, F.; VILLAVERDE, R.; MORENO, V. Y GONZÁLEZ, V. (2000). Caracterización in situ de la variabilidad del mamey colorado Pouteria sapota (Jacq.) Moore et Stearn en dos regiones de Cuba. III Taller Nacional "Contribución de los Huertos Caseros a la conservación in situ de Recursos Fitogenéticos en Sistemas de Agricultura Tradicional" INIFAT, 24-25 enero del 2001. Cuba. 
SHAGARODSKY, T., FUNDORA, Z.; CASTIÑEIRAS, L.; BARRIOS, O.; MORENO, V.; FERNÁNDEZ, L.; FUENTES, V.; CRISTÓBAL, R.; GIRAUDY, C.; GARCÍA, M.; SÁNCHEZ, P.; GONZÁLEZ, V.; FERNÁNDEZ, F.; VALIENTE, A. 2001a. Diversidad de los productos agrícolas en los sistemas de fincas y su presencia en el mercado. Cuba. Agricultura Orgánica No.1 (7): $24-28$.
SHAGARODSKY, T., FUNDORA, Z.; CASTIÑEIRAS, L.; BARRIOS, O.; GIRAUDY, C.; GARCÍA, M.; FERNÁNDEZ, L.; PULDÓN, G.; PÉREZ, M. F.2001b. Inventario de la diversidad de plantas de cultivo en el mercado agrícola. Memorias IV Taller Internacional sobre Recursos Fitogenéticos FITOGEN2001. Estación Experimental de Pastos y Forrajes, Sancti Spíritus, Cuba p. $49-51$. 